\title{
Compressed Sensing of Wireless Signals for Image Tensor Reconstruction
}

Scott Fowler, Gabriel Baravdish and George Baravdish

The self-archived postprint version of this journal article is available at Linköping University Institutional Repository (DiVA):

http:/ / urn.kb.se/ resolve?urn=urn:nbn:se:liu:diva-168209

N.B.: When citing this work, cite the original publication.

Fowler, S., Baravdish, G., Baravdish, G., (2019), Compressed Sensing of Wireless Signals for Image Tensor Reconstruction, 2019 IEEE GLOBAL COMMUNICATIONS CONFERENCE (GLOBECOM). https:// doi.org/ 10.1109/ GLOBECOM38437.2019.9014260

Original publication available at:

https:/ / doi.org/ 10.1109/ GLOBECOM38437.2019.9014260

Copyright: IEEE

http:/ / www.ieee.org/

(C) 2019 IEEE. Personal use of this material is permitted. However, permission to reprint/ republish this material for advertising or promotional purposes or for creating new collective works for resale or redistribution to servers or lists, or to reuse any copyrighted component of this work in other works must be obtained from the IEEE. 


\title{
Compressed Sensing of Wireless Signals for Image Tensor Reconstruction
}

\author{
Scott Fowler ${ }^{1}$, Gabriel G. Baravdish ${ }^{2}$ and George Baravdish ${ }^{3}$ \\ ${ }^{1}$ Communications and Transport Systems, ${ }^{2}$ Media and Information Technology \\ ${ }^{3}$ Physics, Electronics and Mathematics \\ Department of Science and Technology, Linköping University, Norrköping, Sweden
}

\begin{abstract}
Use of wireless signal for identification of unknown object, or technology to see-through a wall to form an image, is gaining growing interest from various fields including law enforcement and military sectors, disaster management, or even in civilian sectors such as construction sites. The great challenge in the implementation of such technology is the stochastic disturbances on wireless signal which will result in a signal with missing samples. Compressive Sensing (CS) is a powerful tool for estimating the missing samples since it can find accurate solution to largely underdetermined linear wireless signals. However, sparse models like CS can also suffer from information loss dues to stochastic lossy nature of wireless, making CS not to have accurate information for reconstruction of a signal. In this paper, we developed a theoretical and experimental framework for the mapping of obstacles by reconstructing the wireless signal based on a sparse signal. We apply tensor format to perform the computations along each mode by relaxing the tensor constraints to obtain accurate results. The proposed framework demonstrates how to take $2 \mathrm{D}$ signals, formulate estimate signals and produce a 3D image location in a completely unknown area inside of the obstacle (wall).
\end{abstract}

Index terms-Wireless signals, Compressed Sensing, Tensors, Augmented-Lagrangian, Alternating Direction Method of Multipliers, Basis Pursuit, Ill-posed and Inverse Problems, Imaging.

\section{INTRODUCTION}

Wireless devices have been used to investigate the environment and understand our physical world. Recently, IoT devices are widely adopted to gather sensory data and reconstruct the environment in the cyberspace [1], [2]. Of particular interest is the use of wireless signals to identify unknown objects behind walls as it can be applied in numerous scenarios where assessing the situation prior to entering an area can be very crucial. For example, sectors like law enforcement and military sectors, disaster management, or even in civilian sectors such as construction sites. Despite the great potential in the technology to see-through a wall to form an image, there is a considerably challenging problems to solve before its wider implementation, namely, interference with everyday radio frequency (RF) signals. Forming an image of unknown objects based on the measurements of scattered electric fields has been an ill-posed problem [3]-[6] for the past several years. Previously proposed methods for solving the inverse scattering problem include use of iterative techniques, contrast source inversion, stochastic optimization. Another approach is the use of WiFi signal to 2D imaging using RSSI [7], [8]. However, raw dataset gathered by wireless devices usually suffer from missing data due to stochastic lossy nature of a wireless signal, which is a great challenge for accurate reconstruction.

Traditionally, retransmission schemes have been applied to address the stochastic disturbances on wireless channel, but they can cause more congestion and round trip delays and not suitable for the current scenarios. Forward error correction schemes like Reed-Solomon [9] are better suited, but the usage of low-power sensing is limited for their computational complexity or bandwidth overhead [10], [11]. In the accurate reconstruction for only a few missing values, the problem can be solved by focusing on the dataset. Such approaches for interpolate missing data include K-Nearest Neighbors (KNN) [12], Delaunay Triangulation (DT) [13], and Multichannel Singular Spectrum Analysis (MSSA) [14]. The drawback is that they cannot be applied when the data loss is too high [15].

Compressive Sensing (CS) [16], [17] is a powerful approach for estimating missing samples. CS framework uses nonlinear reconstruction algorithms to accurately recover the entire signal dataset from measurements. Generally, the number of measurements will only be a small fraction of the original signal dataset as long as the signal data contain sparse/low-rank features. In case of CS, however, each transmitted bit of information is precious on a lossy wireless network. Thus, existing CS-based reconstruction methods require inherent structure for the wireless signal and redundancy for the dataset. In addition, the dataset for CS must be Gaussian or pure random distribution to fill in missing values. Nonetheless, in IoT devices, the missing sample values do not satisfy these prerequisites [15]. An intrinsic limitation in conventional CS theory is that it relies on data representation in the form of vectors. In fact, many signal data types do not lend themselves to vector data representation. Such example are wireless technology, imaging and video processing to just to mention a few [18]. Using standard CS method, one must multiplex the signal data of all dimensions using a global sampling device, which is difficult to implement especially with missing signal data points [19], [20]. Given the challenges in current CS, new technique that can effectively and efficiently estimate the values of the missing points in a signal should be developed. One such promising approach is tensor framework. Tensor framework will provide complete measurements for CS reconstruction algorithm in multidimension. 


\section{Problem Formulation IN 1D Signals}

In this section we give a brief background on reconstructing one dimensional signals. CS theory is based on the sparsity of signal much lower than that of the classical Nyquist sampling [21]. The CS theory states if a signal $s \in \mathbb{R}^{N}$ is sparse, i.e. $\|s\|_{0} \ll N$, then we are able to reconstruct it from any $M$ sample values by an appropriate linear transformation $\Phi$. Conventional CS measurements from its vectorized version via:

$$
y=\Psi x+v,
$$

where $\Psi \in \mathbb{R}^{M \times N}(M<N)$ is the sensing matrix (also called the sampling or measurement matrix), $x \in \mathbb{R}^{N}$ denotes the vectorized signal, $y \in \mathbb{R}^{M}$ signal of interest and $v \in \mathbb{R}^{M}$ represents noise. The vectorized signal is assumed to be sparse in some sparsifying basis $\Phi \in R^{M \times N}$, can be written as:

$$
x=\Phi s,
$$

where $s \in R^{N}$ is the sparse representation of $x$ and it has only $K \ll N$ non-zero coefficients. Thus the sensing model can be rewritten as:

$$
y=\Psi \Phi s+v=A s+v,
$$

where $A=\Psi \Phi \in R^{M \times N}$ is the equivalent sensing matrix. The standard CS theory primarily focuses on one dimensional (1D) signals. According to [22], CS is capable of recovering $K$-sparse signal from $y$ of size $M$, provided that the number of random samples meets $M \geq \alpha K(N / K)$. When the samples rate fall below, the problem is more difficult to solve since in addition to the non-convexity of the loss term, both the loss and regularization terms are non-smooth. Figure 1 illustrates a signal without any missing samples (Top plot: Complete Signal) and a signal with missing samples (Bottom plot: Incomplete Signal). The wireless signal is a 270 time sample measurements from the TX/RX but with 170 samples missing. To clarify, 100 of the 270 samples are known. Due to data transmission errors or acquisition errors, some samples of a signal may be lost. The problem is to fill in the missing 170 samples. In order to conceal these errors, the missing samples must be filled in with suitable values. Filling in missing values in order to conceal errors is called error concealment, which is computational complexity or bandwidth heavy [23]. For these reasons, applying conventional CS to applications requires forming a model for multidimensional signals in a tensor framework, which allows a signal to operate in more than 1D while being able rebuild missing values of a signal.
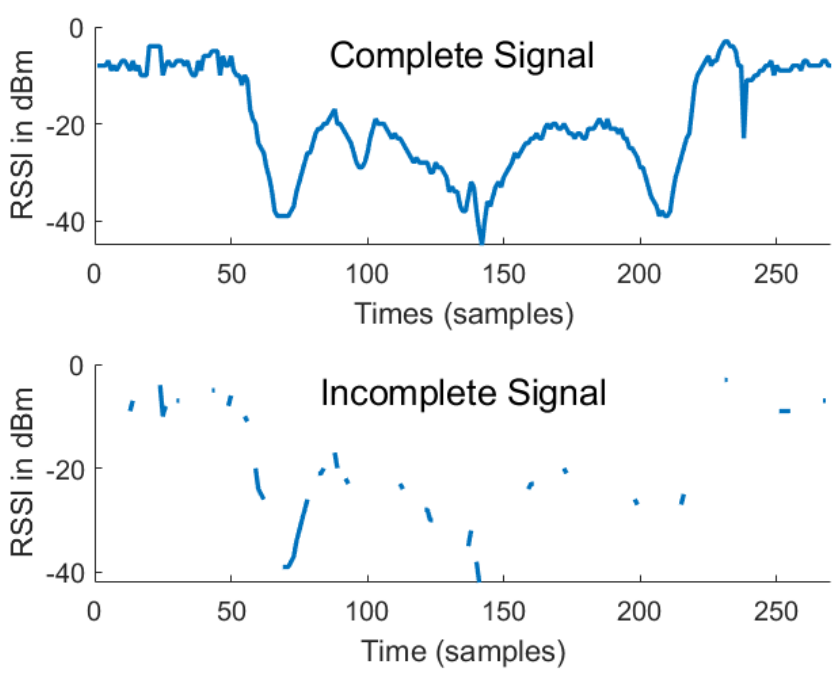

Fig. 1: (Top) Is a Complete Signal with no missing samples. (Bottom) Is the same Signal but with Missing Samples.

The problem of estimating $x$ from $y$ in (1) is called a linear inverse problem (LIP); for most scenarios of practical interest, this is an ill-posed LIP. Given that $x$ is sparse and the sensing matrix $A$ satisfies some stable embedding conditions [16], then it is possible to accurately recover $x$ with an error upper bounded by the noise strength. To reconstruct the sparse structure of $x$ gives us the following optimization problem: 


$$
\min _{s \in \mathbb{R}^{N}}\|s\|_{0} \quad \text { s.t. }\|A s-y\|_{2} \leq \varepsilon,
$$

where $\|s\|_{0}$ is known as $\ell_{0}$-norm and $\varepsilon>0$ bounds $\ell_{2}$-norm defined residual error level. However, when $y$ is noisy, then it is not desired that $y=A s$ be solved exactly. Also, it is not numerically feasible since it is NP-Hard. One way to transition the problem into something more tractable is to replace $\|\cdot\|_{0}$ by relaxing with its convex approximation $\|\cdot\|_{1}$ (e.g. Basis Pursuit (BP)), which leads us to the following:

$$
\min _{x \in \mathbb{R}^{N}}\|s\|_{1} \quad \text { s.t. }\|A s-y\|_{2} \leq \varepsilon .
$$

\section{PROBLEM REFORMULATION IN TENSOR FRAMEWORK}

The inverse problem (1) can be generalized, as formulated in [24], for multi-dimensional signals as:

$$
\mathcal{Y}=\mathcal{X} \times{ }_{1} \Psi_{1} \times_{2} \Psi_{2} \cdots \times_{n} \Psi_{n}+\mathcal{V}
$$

where $\mathcal{Y} \in \mathbb{R}^{M_{1} \times \cdots \times M_{n}}$ represents the measurement, $\mathcal{X} \in \mathbb{R}^{M_{1} \times \ldots \times M_{n}}$ is the signal which to be reconstructed, $\mathcal{V} \in$ $\mathbb{R}^{M_{1} \times \cdots \times M_{n}}$ denotes the noise term and $\Psi_{i} \in \mathbb{R}^{M_{i} \times \cdots \times M_{n}}, i=1, \ldots, n$, is the sensing matrix and $M_{i}<N_{i}$. The multidimensional signal is assumed to be sparse in a $K$ :

$$
\mathcal{X}=\mathcal{S} \times{ }_{1} \Phi_{1} \times_{2} \Phi_{2} \cdots \times_{n} \Phi_{n},
$$

where $\mathcal{S} \in \mathbb{R}^{N_{1} \times \cdots \times N_{n}}$ is the sparse representation that has only $K_{1}, K_{2}, \ldots, K_{n}$ number of non-zero coefficients along each dimension. The similar sensing model could then be:

$$
\mathcal{Y}=\mathcal{S} \times{ }_{1} A_{1} \times{ }_{2} A_{2} \cdots \times{ }_{n} A_{n}+\mathcal{V},
$$

where $A_{i}=\Psi_{i} \Phi_{i}, i=1, \ldots, n$, are the similar sensing matrices. It is also should be noted that the model in (8) is similar to:

$$
y=\left(A_{n} \otimes A_{n-1} \otimes \cdots \otimes A_{1}\right) s+v,
$$

where $\otimes$ represents is Kronecker product. By expressing $\widetilde{A}=A_{n} \otimes A_{n-1} \otimes \cdots \otimes A_{1}$, it becomes a conventional CS model comparable to (3), except the sensing matrix in (9) has a multi-linear structure, i.e., $y=\widetilde{A} s+v$.

We can now reconstruct an $n$-dimensional signal $\mathcal{X}$, given that the signal is $\left(K_{1}, \ldots, K_{n}\right)$-sparse under a set of dictionaries $\left\{\Phi_{1} \in \mathbb{R}^{M_{1} \times K_{1}}, \ldots, \Phi_{n} \in \mathbb{R}^{M_{n} \times K_{n}}\right\}$ by formulating the following constrained minimization problem:

$$
\begin{aligned}
\min _{\mathcal{S}} & \|\mathcal{S}\|_{0} \\
\text { s.t } & \left\|\mathcal{Y}-\mathcal{S} \times{ }_{1} A_{1} \times{ }_{2} \ldots \times{ }_{n} A_{n}\right\|_{F} \leq \varepsilon,
\end{aligned}
$$

where the norm of a tensor $\mathcal{Z} \in \mathbb{R}^{M_{1} \times \ldots \times M_{n}}$ is defined by

$$
\|\mathcal{Z}\|_{F}=\sqrt{\sum_{i=1}^{M_{1}} \sum_{i=1}^{M_{2}} \cdots \sum_{i=1}^{M_{n}} z_{i_{1} i_{2} \cdots i_{n}}^{2}} .
$$

This is analogous to the matrix Frobenius norm for a matrix. Note that in the multi-dimensional case, the measurement matrices act independently along each dimension. However, we can convert (10) to a lower dimensional domain. For the vectorized case, it is formulated as:

$$
\begin{array}{cl}
\min _{s} & \|s\|_{0} \\
\text { s.t. } & \left\|y-\left(A_{n} \otimes A_{n-1} \otimes \cdots \otimes A_{1}\right) s\right\|_{2} \leq \varepsilon .
\end{array}
$$

Finally, as in (5), we recast $\|s\|_{0}$ in (11) by relaxing with its convex approximation $\|s\|_{1}$, giving us:

$$
\begin{array}{cl}
\min _{s} & \|s\|_{1} \\
\text { s.t. } & \left\|y-\left(A_{n} \otimes A_{n-1} \otimes \cdots \otimes A_{1}\right) s\right\|_{2} \leq \varepsilon .
\end{array}
$$




\section{Proposed Sparsity Model Coefficient using BP}

In this section we recast the (12) into the following minimization unconstrained problem:

$$
\min _{s}\|s\|_{1}+\frac{\gamma}{2}\left\|y-\left(A_{n} \otimes A_{n-1} \otimes \cdots \otimes A_{1}\right) s\right\|_{2}^{2},
$$

where $\gamma>0$. To manage the mapping of the tensor format to multi-linear system, we express it as $\widetilde{A}=A_{n} \otimes A_{n-1} \otimes \cdots \otimes A_{1}$. While it is possible to keep the system in full tensor format and thus perform the computations along each mode, we were able to still obtain the accurate results by relaxing full mapping of the tensor format. Also, being that $\|\cdot\|_{1}$ is defined as the sum of absolute values are not differentiable since the absolution value function is not differentiable at zero. The fact that the cost functions are non-differentiable makes solving the problem somewhat difficult, especially for large-scale problems.

\section{A. Variable Split}

The approach in this paper is based on variable splitting, which goes back at least to the 40's [25], have been recently used to address large-scale optimization problems. The main idea consist in splitting the variable of interest $x$ an introduce an additional optimization variable $z$ and then solving the counterpart minimization problem in the the form:

$$
\min _{x, z} f(x)+g(z) \text { s.t. } x=z,
$$

where $f$ is continuously differentiable and $g$ stands for some regularization function which is convex. By splitting the objective function in (14), it offers a general framework for solving regularization problems while filling in missing values.

\section{B. Augmented-Lagrangian}

In general, the method of splitting the optimization problem into subproblems which are either to addressed both unconstrained and constrained or for easy in solving the problem is known as Augmented-Lagrangian (AL) [26], specifically the alternating direction method of multipliers (ADMM) [27], [28]. Is suitable is solving a broad class of $\|\cdot\|_{1}$ regularized problems [29]. Despite AL is known to be comparable to Bregman, which was proposed to address constrained optimization formulations for solving inverse problems (refer to [30] and references therein), we use AL viewpoint instead of Bregman iterative view, since it is a more standard/elementary tool (covered in most optimization textbooks). The AL is obtained by associated with (14):

$$
\mathcal{L}_{A}(x, z, \xi, \eta)=f(x)+g(z)+\xi^{T}(x-z)+\frac{\eta}{2}\|x-z\|_{2}^{2},
$$

where $\xi$ is Lagrange multiplier and $\eta>0$ is called the penalty parameter. The ADMM solves (14) via an iteration which entails minimization of $\mathcal{L}_{A}(x, z, \xi, \eta)$ independently on $x$ and $z$, plus a gradient ascent update of $\xi$ until some convergence criterion is satisfied. Note, AL function is not the same as the standard Lagrangian function, it includes a square penalty term, and differs from the quadratic penalty function by the fact of the exists of the linear term involving the multiplier $\xi$. In other words, AL is a combination of the Lagrangian and quadratic penalty functions.

\section{Estimating Missing Sparsity Data}

Due to the stochastic lossy natural of a wireless signal, errors occur from transmission or acquisition of a signal, some samples possible be lost. In order to address the problem, the missing samples must be filled in with proper values. Given the incomplete signal $y$, we need to find $x$ such that $y=\Psi x$. Now suppose the signal $x$ has a sparse representation with respect to $\Phi$, meaning that $x$ can be represented as:

$$
x=\Phi s,
$$

where $s$ is a sparse coefficient vector of length $N$ and $\Phi$ is a matrix of size $M \times N,(M \leq N)$. The incomplete signal $y$ can then be written as $y=\Psi x$. Therefore, if we can find a $s$ based on (16) which satisfying:

$$
y=\Psi \Phi s=A s,
$$

then we can create an estimate $\hat{x}$ of $x$ by setting $\hat{x}=\Phi s$.

From (17) it is clear that $\Psi \hat{x}=y$ therefore, $\hat{x}$ agrees with the known samples of $x$. The signal $\hat{x}$ is the same length as $x$. Being that $y$ is shorter than the coefficient vector $s$, so there are infinitely many solutions to (17). Any set of $s$ values satisfying $y=\Psi \Phi s=A s$ can be considered a valid set of coefficients.

To estimate the missing values and deal with the problem (14) is by:

$$
\begin{aligned}
\min _{s, z} & \overbrace{\frac{1}{2}\|y-A s\|_{2}^{2}}^{f(s)}+\overbrace{\lambda\|z\|_{1}}^{g(z)} \\
\text { s.t. } & s=z .
\end{aligned}
$$


By alternating minimization with respect to $f(s)$ and $g(z)$ allows recovery of missing sample data points. The rationale of these subproblem method is that each step repeating of this alternating minimization may be much easier than the original unconstrained variable split problem (15). When $g(z)$ is the $\ell_{1}$-norm, $\|z\|_{1}=\sum\left|z_{i}\right|$ the proximal operator is determined by soft-thresholding, $\hat{x}=\operatorname{prox}_{\lambda\|\cdot\|_{1}}=\operatorname{sign}(y) \max \{|y|-\lambda, 0\}$. For additional information, see [31]. From here the basic idea is to iterate until the missing samples meet a condition level or until the iteration is completed. In each iteration, a smoothening activation function is applied by means of a threshold. By applying threshold $(\boldsymbol{p r o x}(\cdot))$, we obtain an unbiased estimating the unknown signal $\hat{x}$, based on the given observation of $y$, which consists within the given constrained optimization problem for filling in the missing signal values.

\section{Forming of the $3 D$ Image from $2 D$ Signal}

General term that refers to any reduction in the strength of a signal is referred to attenuation. We are interested in the attenuation in the strength of the signal because inform us about the present of an object along the signal. When an object in present, we expect that the signal which pass through that object will, on average, experience higher shadowing losses (Shadowing loss due to moving objects that attenuate the signal.). The attenuation field is the link shadowing as a basic linear form combination of the values in vector and matrix. By using (3) and (9) our sensing model as:

$$
\Delta y=A \Delta \hat{x}+v,
$$

where $\Delta y$ is the vector of length $M$ of all the links different RSS measurements, $\Delta \hat{x}$ is the attenuation to be estimated and $[\mathbf{A}]_{i j}=w_{i j}$ is the weighting matrix. From the reconstructed estimated received signal, the noise is removed by using a General Gaussian Filter, giving us $\Delta y$. For more details about Gaussian Filtering, see [32].

Next we show how the signal measurements can build a map of extract obstacles. More specifically, we formulate the cleaned signal into a 2D map of the extracted obstacle into 3D map. Let $\Delta y^{\theta}$ represent the 4 sample routes $0^{\circ}, 45^{\circ}, 90^{\circ}$ and $135^{\circ}$ in vector form. The 4 sample routes cover a complete unknown area, for more details we refer to [7], [8], [32]. The vector form of each route is given by $\Delta y^{\theta} \in \mathbb{R}^{M \times 1}, \theta \in\left\{0^{\circ}, 90^{\circ}\right\}$ and $\Delta y^{\theta} \in \mathbb{R}^{1 \times M}, \theta \in\left\{45^{\circ}, 135^{\circ}\right\}$.

If the IoT device has knowledge of an environment, it would be possible to estimate the weights $\left(w_{i j}\right)_{j}$. Having such knowledge, one would be able to also estimation of the linear transformation $A$. However, it is doubtful to have specific information. Therefore we depend on a statistical model to give us a linear outcome of the attenuation on the path loss. The shadowing loss of attenuating objects is modeled as:

$$
\Delta y_{M}^{\theta}=\sum_{j=1}^{M} w_{j} \Delta y_{j}^{\theta}(t),
$$

where $\Delta y_{j}^{\theta}$ is the normal path loss occurring in the signal domain $j$ at time $t$ without attenuation, and $w_{i j}$ is the weight of signal $j$. The weights are calculated as follows:

$$
\omega_{x}= \begin{cases}1, & \text { if } \Delta y_{M}<y_{d B} \\ 0, & \text { otherwise }\end{cases}
$$

where $y_{d B}$ defines the threshold of the desired information.

Next we convert the vectors in (20) into a matrix by repeating the elements in a vector, giving us $\Delta y^{\theta} \in \mathbb{R}^{M \times M}, \theta=$ $\left\{0^{\circ}, 45^{\circ}, 90^{\circ}, 135^{\circ}\right\}$.

Occluded cylinder structure of interest that is completely unknown and needs to be imaged. Now we layer the matrix on top of each other of the unknown area to be imaged:

$$
\operatorname{Img}\left(\Delta y_{M \times M}^{0^{\theta}, \ldots, 135^{\theta}}\right)=\left\{\begin{array}{cl}
\Delta y_{M \times M}^{0^{\theta}, \ldots, 135^{\theta}}, & \text { if } \Delta y_{M \times M}^{0^{\theta}, \ldots, 135^{\theta}}>\delta \\
0, & \text { otherwise, }
\end{array}\right.
$$

where $\delta$ is the thresholds. The object can be at any point with equal probability in the link's effective region, hence the $\delta$ provides the pruning in each $M \times M$ stages.

\section{RESULTS}

The study assumes that reconstruction is solely based on wireless measurements generated from the Gaussian filter and used occlude cylinder as unknown subject model. In Figures 2, 3 and 4, we show the graphical representation of the reconstructed images of the occluded cylinder behind the wall. Our proposed CS-based signal reconstruction is based on the data set presented in [7], [8] $]^{1}$. We validated the performance of proposed algorithm under different sparsity by reconstructing the sparse signal from missing samples, and constructed 3D imaging of the occluded cylinder in the unknown area (behind the walls).

\footnotetext{
${ }^{1}$ Data set available at: http://dx.doi.org/10.21229/M9RP4K
} 
Performance validation of the proposed algorithm: Figure 2 is the graphical comparison between the images from the complete measurement of the CS reconstruction and the incomplete signals. The one on the left-hand side represents the image resulted from missing samples (incomplete signals), which is scattered and the shape is not identifiable. The right-hand side image represents the result of reconstructed signals, from which one can clearly identify the 3D shape of the subject (cylinder). In the test, the full signal length consists of 250 to 270 samples, and the number of known signal samples among that is 100 , which is approximately $35 \%$ to $40 \%$ of total samples. The results were quite similar when the number of known samples was reduced in other tests.

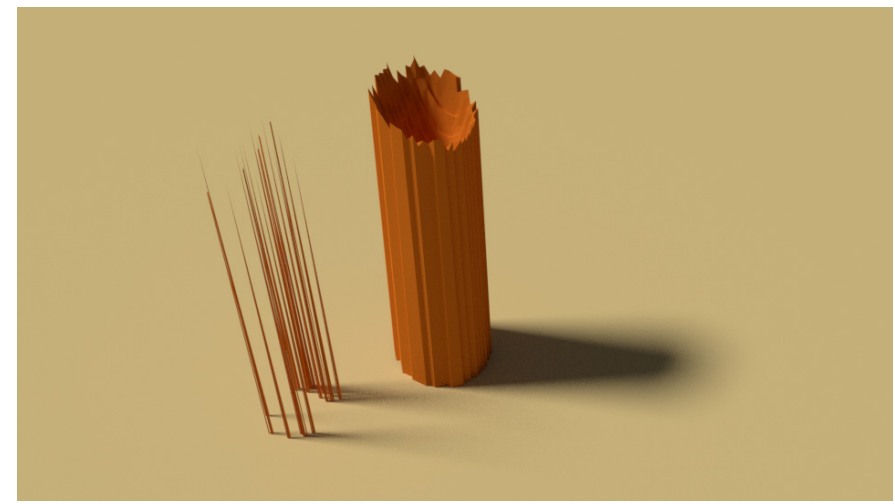

Fig. 2: A 3D comparison with the incomplete signal (left) and the reconstructed signal of the occluded cylinder (right).

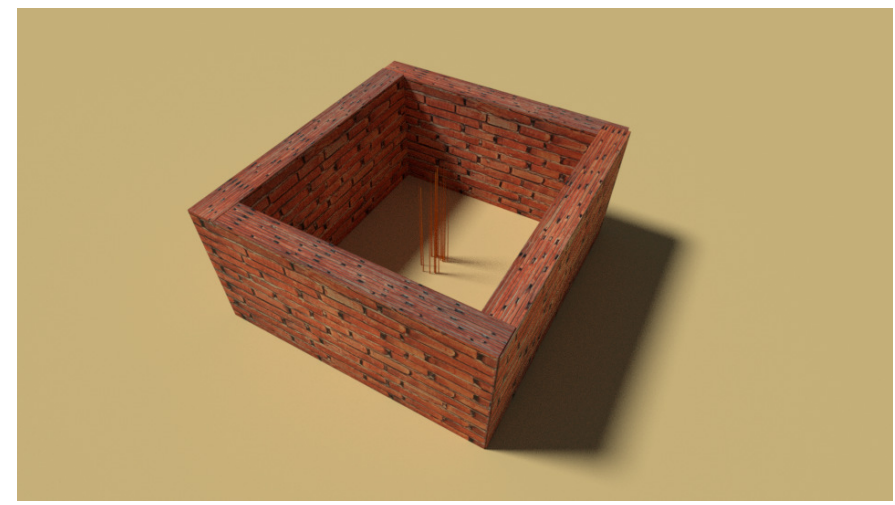

Fig. 3: Shows a 3D imaging of the signal with missing samples, represented as an incomplete cylinder in the unknown area. The area in this case is behind the walls.

Figures 3 and 4 illustrate the same test results when the subjects are occluded behind the wall. While in the incomplete signal image, Figure 3, the occluded object behind the wall is unidentifiable, the CS reconstruction image, Figure 4, the cylinder shape is successfully restored in unknown area.

Plot of the estimation of missing signal values using our method: To obtain the solution to the BP-problem (5), we have used 100 iterations of an iterative algorithm. Note, the iterative estimated algorithm converged between 30-45 iterations as it reaches the minimum value. The coefficient $s$ obtained by solving the estimated signal $\hat{x}=\Phi s$. As shown in Figure 2 , the imaging quality is considerably high with the estimation of missing values and the shape of the occluded object can be clearly identified as a cylinder, unlike the construction with incomplete signals. The presence of a dip in the center (Figure 2, right) indicates the occluded cylinder is hollow. Even with minimal wireless measurements, our estimated signal reconstruction can build the original structures in 3D image and restore their details features such as height, location in a completely unknown workspace (Figure 4).

Relative error with increased number of missing samples against the complete signal: In Figures 5 and 6 the $\mathrm{x}$-axis represents sparsity, i.e. the proportion of the missing samples of the original signal. The y-axis represents the relative error, which shows how large the error is in the size of the original signal. As the proportion of missing samples increases, the relative error becomes higher for the estimated signal. We conclude that a signal with over $80 \%$ missing samples is the limit of the method to reconstruct with accuracy s due to the high sparsity and too few samples, whereas the signal sparsity below $80 \%$ is the minimum optimal for an estimated signal accuracy as the error is stable and low this range. 


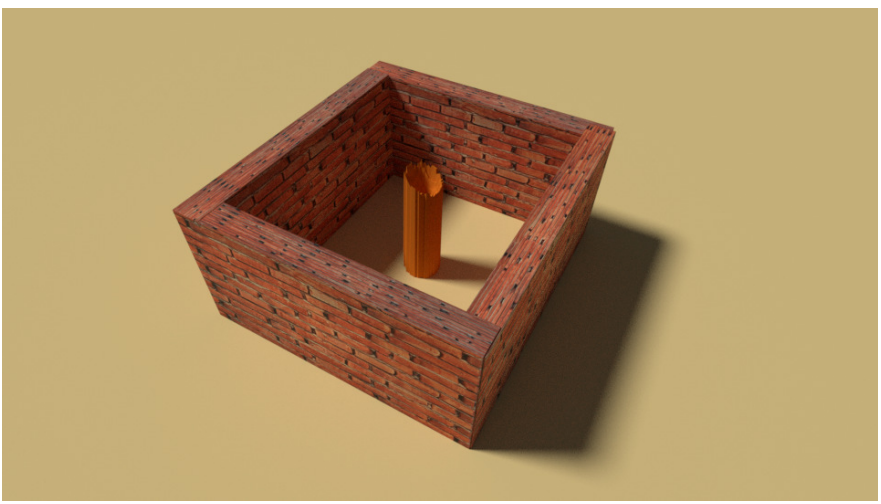

Fig. 4: A 3D image of the reconstructed signal inside an unknown area.

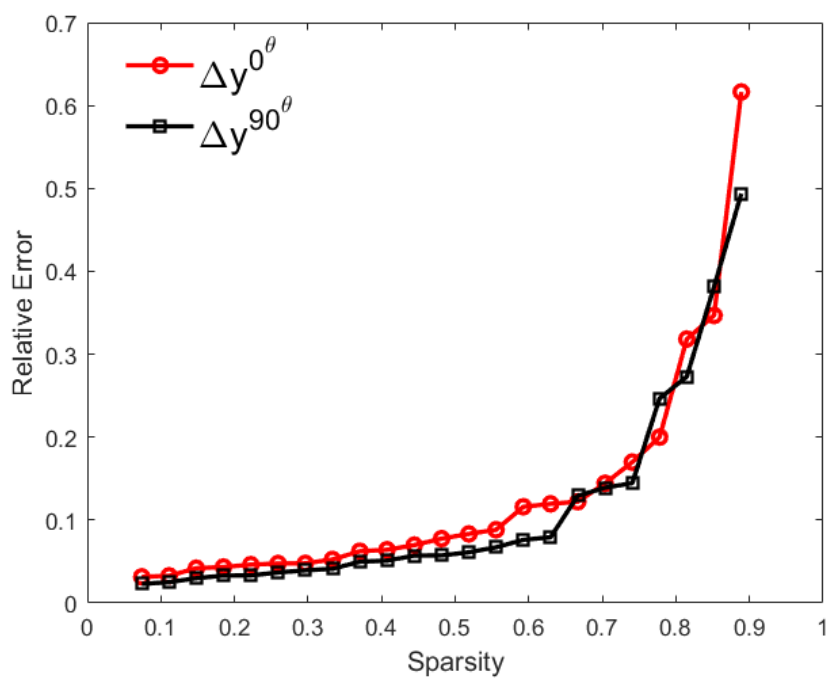

Fig. 5: A graph which represents the relative error against sparsity for the estimated signal and the original signal. The sparsity represents the proportion of missing samples of the original data. The graph results starts at 7\% missing samples up to $90 \%$ missing samples.

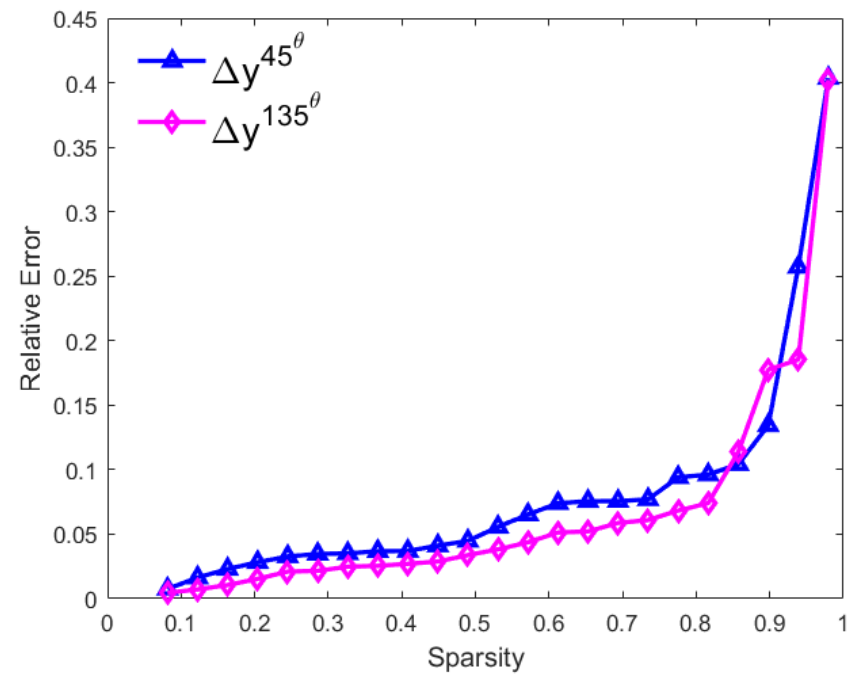

Fig. 6: The relative error for the estimated signal against its sparsity. Here, the sparsity starts at $8 \%$ missing samples up to $97 \%$ missing samples. 


\section{CONCLUSION}

Reconstruction of signal can become a great challenge due to the missing samples from a signal caused by node failures and interference. In this work, we focused on the formulation of tensor aspects algorithm to reconstruction of signal. While the simple complete tensor format allows the computations along each mode, we achieved the accurate results with AugmentedLagrangian, namely, alternating direction method of multipliers by relaxing the tensor constraints. We demonstrated methods to take 2D signal, formulate estimate signal, and produce a 3D image location in a completely unknown area inside of the obstacle (wall) or workplace. Future work will investigate different sparse representation dictionaries, including dictionary learning, for identification of more occlude objects and human subjects.

\section{ACKNOWLEDGMENT}

The work of Dr. Scott Fowler has been supported by the strategic innovation programme Smart Built Environment, funded by Vinnova, Formas and Energimyndigheten.

\section{REFERENCES}

[1] Tian He, Sudha Krishnamurthy, John A. Stankovic, Tarek Abdelzaher, Liqian Luo, Radu Stoleru, Ting Yan, Lin Gu, Jonathan Hui, and Bruce Krogh. Energy-efficient surveillance system using wireless sensor networks. In Proceedings of the 2Nd International Conference on Mobile Systems, Applications, and Services, MobiSys '04, pages 270-283, New York, NY, USA, 2004. ACM.

[2] O. Elijah, T. A. Rahman, I. Orikumhi, C. Y. Leow, and M. N. Hindia. An overview of internet of things (iot) and data analytics in agriculture: Benefits and challenges. IEEE Internet of Things Journal, 5(5):3758-3773, Oct 2018.

[3] L. C. Potter, E. Ertin, J. T. Parker, and M. Cetin. Sparsity and compressed sensing in radar imaging. Proceedings of the IEEE, 98(6):1006-1020, June 2010.

[4] David Colton and Andreas Kirsch. A simple method for solving inverse scattering problems in the resonance region. Inverse Problems, 12(4):383-393, Aug 1996.

[5] Y. M. Wang and Weng Cho Chew. An iterative solution of the two-dimensional electromagnetic inverse scattering problem. Int. J. Imaging Systems and Technology, 1:100-108, 1989.

[6] N. Joachimowicz, C. Pichot, and J. P. Hugonin. Inverse scattering: an iterative numerical method for electromagnetic imaging. IEEE Transactions on Antennas and Propagation, 39(12):1742-1753, Dec 1991.

[7] S. Depatla, L. Buckland, and Y. Mostofi. X-Ray Vision with Only WiFi Power Measurements Using Rytov Wave Models. IEEE Transactions on Vehicular Technology, 64:1376-1387, April 2015.

[8] Y. Mostofi. Cooperative Wireless-Based Obstacle/Object Mapping and See-Through Capabilities in Robotic Networks. IEEE Transactions on Mobile Computing, January 2012.

[9] I. Reed and G. Solomon. Polynomial codes over certain finite fields. Journal of the Society for Industrial and Applied Mathematics, 8(2):300-304, 1960.

[10] Z. Charbiwala, S. Chakraborty, S. Zahedi, T. He, C. Bisdikian, Y. Kim, and M. B. Srivastava. Compressive oversampling for robust data transmission in sensor networks. In 2010 Proceedings IEEE INFOCOM, pages 1-9, March 2010.

[11] M. Rudelson and R. Vershynin. Sparse reconstruction by convex relaxation: Fourier and gaussian measurements. In 200640 th Annual Conference on Information Sciences and Systems, pages 207-212, March 2006.

[12] T. Cover and P. Hart. Nearest neighbor pattern classification. IEEE Transactions on Information Theory, 13(1):21-27, January 1967.

[13] L. Kong, D. Jiang, and M. Wu. Optimizing the spatio-temporal distribution of cyber-physical systems for environment abstraction. In 2010 IEEE $30 t h$ International Conference on Distributed Computing Systems, pages 179-188, June 2010.

[14] H. Zhu, Y. Zhu, M. Li, and L. M. Ni. Seer: Metropolitan-scale traffic perception based on lossy sensory data. In IEEE INFOCOM 2009, pages 217-225, April 2009.

[15] L. Kong, M. Xia, X. Liu, G. Chen, Y. Gu, M. Wu, and X. Liu. Data loss and reconstruction in wireless sensor networks. IEEE Transactions on Parallel and Distributed Systems, 25(11):2818-2828, Nov 2014.

[16] E. J. Candes, J. Romberg, and T. Tao. Robust uncertainty principles: exact signal reconstruction from highly incomplete frequency information. IEEE Transactions on Information Theory, 52(2):489-509, Feb 2006.

[17] D. L. Donoho. Compressed sensing. IEEE Transactions on Information Theory, 52(4):1289-1306, April 2006.

[18] R. Zhao, J. Fu, L. Ren, and Q. Wang. Atom-refined multiway greedy algorithm for tensor-based compressive sensing. IEEE Access, 7:23038-23054, 2019.

[19] G. R. Arce, D. J. Brady, L. Carin, H. Arguello, and D. S. Kittle. Compressive coded aperture spectral imaging: An introduction. IEEE Signal Processing Magazine, 31(1):105-115, Jan 2014.

[20] M. F. Duarte, M. A. Davenport, D. Takhar, J. N. Laska, T. Sun, K. F. Kelly, and R. G. Baraniuk. Single-pixel imaging via compressive sampling. IEEE Signal Processing Magazine, 25(2):83-91, March 2008

[21] E. J. Candes and M. B. Wakin. An introduction to compressive sampling. IEEE Signal Processing Magazine, 25(2):21-30, March 2008.

[22] E. J. Candes and T. Tao. Near-optimal signal recovery from random projections: Universal encoding strategies? IEEE Transactions on Information Theory, 52(12):5406-5425, Dec 2006.

[23] Yao Wang and Qin-Fan Zhu. Error control and concealment for video communication: a review. Proceedings of the IEEE, 86(5):974-997, May 1998.

[24] L. Kong, M. Zhao, X. Liu, J. Lu, Y. Liu, M. Wu, and W. Shu. Surface coverage in sensor networks. IEEE Transactions on Parallel and Distributed Systems, 25(1):234-243, Jan 2014.

[25] R. Courant. Variational methods for the solution of problems of equilibrium and vibrations. Bull. Amer. Math. Soc., 49(1):1-23, 01 1943.

[26] Jorge Nocedal and Stephen J. Wright. Numerical Optimization. Springer, New York, NY, USA, second edition, 2006.

[27] Jonathan Eckstein and Dimitri P. Bertsekas. On the douglas-rachford splitting method and the proximal point algorithm for maximal monotone operators. Math. Program., 55(3):293-318, June 1992.

[28] Stephen Boyd, Neal Parikh, Eric Chu, Borja Peleato, and Jonathan Eckstein. Distributed optimization and statistical learning via the alternating direction method of multipliers. Found. Trends Mach. Learn., 3(1):1-122, January 2011.

[29] Tom Goldstein and Stanley Osher. The split bregman method for 11-regularized problems. SIAM J. Img. Sci., 2(2):323-343, April 2009.

[30] Wotao Yin, Stanley Osher, Donald Goldfarb, and Jérôme Darbon. Bregman iterative algorithms for $\ell_{1}$-minimization with applications to compressed sensing. SIAM J. Imaging Sciences, 1:143-168, 2008.

[31] Neal Parikh and Stephen Boyd. Proximal algorithms. Found. Trends Optim., 1(3):127-239, January 2014.

[32] Scott Fowler, George Baravdish, and Martin Rudberg. Optimizing compressed sensing for seeing through walls based on wireless signals. In IEEE Symposium on Computers and Communications (ISCC), pages 1-6, June-July 2019. 